\title{
Review of: "Acute Cerebellitis Associated With Anti-homer 3 Antibodies: A Rare Case Report and Literature Review"
}

\author{
Francesc Graus ${ }^{1}$ \\ 1 IDIBAPS August Pi i Sunyer Biomedical Research Institute
}

Potential competing interests: Dr. Graus holds a patent licensed to Euroimmun for the use of IgLON5 in an autoantibody test, for which he receives royalties, and receives honoraria from MedLink Neurology for his role as associate editor.

Neuronal antibodies are increasingly described in patients with non-paraneoplastic cerebellar ataxia supporting an autoimmune pathogenesis which justifies the use of immunotherapy to improve the symptoms. With the exception of antibodies against glutamic acid decarboxylase (GAD) or the metabotropic glutamate receptor 1 subunit (mGluR1) the remaining antibodies have been described in a few patients with cerebellar ataxia and therefore the clinical profile and response to immunotherapy of these patients is not well known (Table 1).

Table 1. Neuronal antibodies in non-paraneoplastic cerebellar ataxia

\begin{tabular}{|c|c|c|}
\hline Antibody & Clinical features & Comments \\
\hline GAD & $60 \%$ chronic progressive course. Concomitant symptoms of SPS in $23 \%$ & Partial response to immunotherapy. \\
\hline mGluR1 & Subacute cerebellar syndrome & Remote history of Hodgkin disease \\
\hline ARHGAP26* & May associate with polyneuropathy & Poor response to immunotherapy \\
\hline ITPR-1 & $\begin{array}{l}\text { Variable clinical manifestation. Only } \sim 40 \% \text { present with cerebellar } \\
\text { ataxia }\end{array}$ & Cancer association in $~ 30 \%$ of patients \\
\hline Neurochondrin & Chronic cerebellar/brainstem syndrome & No improvement after immunotherapy \\
\hline CASPR2 & Paroxysmal ataxic episodes lasting minutes to hours & $\begin{array}{l}\text { Ataxia may antedate the onset of } \\
\text { encephalitis }\end{array}$ \\
\hline Sez6l2 & Subacute onset then stabilization & Associated with parkinsonism \\
\hline AP3B2 & Progressive gait ataxia & Ataxia may have a sensory origin \\
\hline Septin-5 & Subacute cerebellar syndrome & One patient spontaneously recovered \\
\hline GluK2 & Acute cerebellitis & Usually associated with encephalitis \\
\hline Homer-3 & Subacute cerebellar syndrome & Partial improvement after immunotherapy \\
\hline
\end{tabular}

* Also known as GRAF: GTPase regulator associated with focal adhesion kinase. AP3B2: Adaptor protein 3 subunit B2; ARHGAP26: Rho GTPase activating protein 26; CASPR2:contactin-associated protein-like 2; GAD: glutamic acid decarboxylase; GluK2: Glutamate kainate receptor 2; ITPR-1: inositol 1,4,5 triphosphate receptor type 1;mGluR1: metabotropic glutamate receptor type 1; Sez6l2: seizure related 6 homolog like 2.

Homer 3 is a scaffolding protein of the post-synaptic density which is expressed in the dendritic spines of the Purkinje cells of the cerebellum. Homer 3 binds to the C-termini of mGluR1 modulating its functional activity. Homer 3 also binds to inositol 1,4,5 triphosphate receptor type 1 (ITPR1). The physical linkage of group mGluR1 to ITPR1 via Homer 3 is physiologically important for postsynaptic calcium responses to mGluR1 stimulation. Interesting both mGluR1 and ITPR1 are also targets of antibodies associated with cerebellar ataxia. 
The paper of Miao and colleagues presents a new patient with cerebellar ataxia and Homer 3 antibodies that responded to immunotherapy and anti-viral treatment. Authors do not explain why ganciclovir and acyclovir was used as part of the treatment. Authors also comment that after treatment the patient was "obviously improved". The statement implies that cerebellar ataxia associated with Homer 3 antibodies usually improves after immunotherapy. However, as seen in cerebellar ataxias with other antibodies against intracellular antigens, relevant improvement in anti-Homer 3 cerebellar ataxia is unusual and, in any case, only partial.

The paper also has other mistakes. 1) In the introduction authors fail to mention that the first case description of Homer 3 antibodies was in 2007 and not in 2013 as they say. The 2013 paper reported the second patient. 2) Authors failed to included in the literature review the third case described (Xu X, Ren H, Li L, Wang J, Fechner K, Guan H. J Neuroimmunol. 2019 May 15;330:155-158). 3) In the discussion authors say that "Guan et al. screened the serum..." no reference is include. In fact, the author's name is not Guan but M. Liu and the reference is number 3 of the paper. 4) Also in the discussion, authors say that Figure 1A "was depicted by a female patient with anti-NMDAR encephalitis"! and they quote a reference (number 9) that should be removed because it is not relevant to the main aim of the paper. In fact this paragraph should be moved to acknowledgements.

In my opinion the flaws of this paper are not only responsibility of the authors but also of the Editor and the reviewers that failed to pick up these obvious mistakes. Frontiers in Neurology should carefully check this issue. 